\title{
Globally consistent 3D mapping with scan matching
}

\author{
Dorit Borrmann, Jan Elseberg, Kai Lingemann, Andreas Nüchter*, Joachim Hertzberg \\ University of Osnabrück, Institut of Computer Science, Knowledge-Based Systems Research Group, Albrechtstreet 28, D-49069 Osnabrück, Germany
}

Received 25 April 2007; received in revised form 11 July 2007; accepted 12 July 2007

Available online 18 July 2007

\begin{abstract}
A globally consistent solution to the simultaneous localization and mapping (SLAM) problem in 2D with three degrees of freedom (DoF) poses was presented by Lu and Milios [F. Lu, E. Milios, Globally consistent range scan alignment for environment mapping, Autonomous Robots 4 (April) (1997) 333-349]. To create maps suitable for natural environments it is however necessary to consider the 6DoF pose case, namely the three Cartesian coordinates and the roll, pitch and yaw angles. This article describes the extension of the proposed algorithm to deal with these additional DoFs and the resulting non-linearities. Simplifications using Taylor expansion and Cholesky decomposition yield a fast application that handles the massive amount of 3D data and the computational requirements due to the 6DoF. Our experiments demonstrate the functionality of estimating the exact poses and their covariances in all $6 \mathrm{DoF}$, leading to a globally consistent map. The correspondences between scans are found automatically by use of a simple distance heuristic.
\end{abstract}

(c) 2007 Elsevier B.V. All rights reserved.

Keywords: Simultaneous localization and mapping (SLAM); 6D SLAM; GraphSLAM; Scan matching

\section{Introduction}

A globally consistent representation of a robot's environment is crucial for basic robot tasks such as localization and navigation. Equipped with a laser scanner, many mobile systems gather information about their local environments. These local representations have to be matched to build a global map. Iterative application of pairwise matching algorithms leads to inconsistencies due to errors in laser scans and the matching procedures itself. To avoid these problems, global matching algorithms are needed, taking correspondences between all scans into account. The common methods for merging all scans are based on probabilistic information. Lu and Milios presented a solution using a network of relations between laser scan poses. A single linear equation system, built from all error measurements, yields optimal estimations for all poses. Limited to 2D laser scans, this approach does not fulfil the requirements for building a correct map of outdoor environments. In this article we extend the linear estimation algorithm to work with 3D scans and 6DoF. To run the algorithm automatically,

\footnotetext{
* Corresponding author. Tel.: +495419692623.

E-mail addresses: nuechter@informatik.uni-osnabrueck.de, andreas@nuechti.de (A. Nüchter).
}

the network is built using a distance heuristic. Furthermore, we integrate the global optimization into a system consisting of odometry extrapolation and the well-known iterative closest point algorithm (ICP). After describing the problem formulation and the algorithm we document its functionality by experimental results.

\section{Related work}

\subsection{Categorization of robotic mapping algorithms}

One way to categorize mapping algorithms is by the map type. The map can be topological or metrical. Metrical maps represent quantitative distances of the environment. These maps can either be $2 \mathrm{D}$, usually an upright projection, or $3 \mathrm{D}$, i.e., a volumetric environment map. Furthermore, SLAM approaches can be classified by the number of DoFs of the robot pose. A $3 \mathrm{D}$ pose estimate contains the $(x, y)$-coordinate and a rotation $\theta$, whereas a $6 \mathrm{D}$ pose estimate considers all DoFs a rigid mobile robot can have, i.e., the $(x, y, z)$-coordinate and the roll, yaw and pitch angles.

In the literature, three different techniques are used for generating 3D maps: First, a planar localization method combined with a 3D sensor; second, a precise $6 \mathrm{D}$ pose estimate 
combined with the data from a 2D sensor; and third, a 3D sensor with a $6 \mathrm{D}$ localization method. Table 1 summarizes these mapping techniques (non-shaded) in comparison with planar 2D mapping (shaded). In this paper, we focus on 3D data and 6D localization, hence on 6D SLAM.

\subsection{Planar $2 D$ mapping}

The state of the art for planar 2D metric maps is probabilistic methods, where the robot has probabilistic motion and uncertain perception models. By integrating these two distributions with a Bayes filter, e.g., a Kalman or particle filter, it is possible to localize the robot. Mapping is often an extension to this estimation problem. Beside the robot pose, positions of landmarks are estimated. Closed loops, i.e., a second encounter of a previously visited area of the environment, play a special role here. Once detected, they enable the algorithms to bound the error by deforming the already mapped area such that a topologically consistent model is created. However, there is no guarantee for a correct model. Several strategies exist for solving SLAM. Thrun reviews in [29] existing techniques, i.e., maximum likelihood estimation [12], expectation maximization [10,30], extended Kalman filter [9] or (sparse extended) information filter [33]. In addition to these methods, FastSLAM [32], which approximates the posterior probabilities, i.e., robot poses, by particles, and the method of Lu/Milios on the basis of IDC scan matching [20], play an important role in $2 \mathrm{D}$.

\subsection{Planar $3 D$ mapping}

Instead of using 3D scanners, which yield consistent 3D scans in the first place, some groups have attempted to build $3 \mathrm{D}$ volumetric representations of environments by translating 2D laser range finders. Thrun et al. [32], Früh et al. [13] and Zhao et al. [41] use two 2D laser scanners for acquiring 3D data. One scanner is mounted horizontally, the other vertically. The latter one grabs a vertical scan line which is transformed into 3D points based on the current 3D robot pose. Since the vertical scanner is not able to scan sides of objects, Zhao et al. use two additional, vertically mounted 2D scanners, shifted by $45^{\circ}$ to reduce occlusions [41]. The horizontal scanner is used to compute the $3 \mathrm{D}$ robot pose. The precision of $3 \mathrm{D}$ data points depends, besides on the precision of the scanner, critically on that pose estimation.

Recently, different groups have employed rotating SICK scanners for acquiring 3D data $[18,28,39]$. Wulf et al. let the scanner rotate around the vertical axis. They acquire 3D data while moving, thus the quality of the resulting map crucially depends on the pose estimate that is given by inertial sensors, i.e., gyros [39]. In addition, their SLAM algorithms do not consider all six DoFs.

\subsection{Slice-wise 6D SLAM}

Local 3D maps built by translated 2D laser scanners and 6D pose estimates are often used for mobile robot navigation. A well-known example is the grand challenge, where the
Stanford racing team used this technique for high speed terrain classification [34].

\subsection{Full 6D SLAM}

A few other groups use highly accurate, yet somewhat immobile 3D laser scanners [1,14,27]. The RESOLV project aimed at modeling interiors for virtual reality and telepresence [27]. They used a RIEGL laser range finder on robots and the ICP algorithm for scan matching [5]. The AVENUE project develops a robot for modeling urban environments [1], using a CYRAX scanner and a feature-based scan matching approach for registering the 3D scans. However, in their recent work they do not use data of the laser scanner in the robot control architecture for localization [14]. The group of $\mathrm{M}$. Hebert has reconstructed environments using the Zoller+Fröhlich laser scanner and aims to build 3D models without initial position estimates, i.e., without odometry information [15]. Magnusson and Duckett proposed a 3D scan alignment method that - in contrast to the previously mentioned research groups - does not use the ICP algorithm, but the normal distribution transform instead [21].

In principle, the probabilistic methods from planar 2D mapping are extendable to 3D mapping with 6D pose estimates. However, no reliable feature extraction nor a strategy for reducing the computational costs of multi hypothesis tracking, e.g., FastSLAM, that grows exponentially with the DoF, has been published to our knowledge.

\subsection{Recent trends}

A recent trend in SLAM research is to apply probabilistic methods to 3D mapping. Katz et al. use a probabilistic notion of ICP scan matching [17]. Weingarten et al. [37] and Cole et al. [8] use an extended Kalman filter on the mapping problem. In the present paper, we extend this state of the art by a GraphSLAM method. A similar approach was used in [35]. However, their algorithm is not practical due to the reported computational requirements. Without considering any sensor data such as laser scans, Olson et al. create globally consistent maps by minimizing the global non-linear constraint network on a set of poses [24]. Triebel et al. apply this approach to their multi-level surface maps [36]. Furthermore, Frese presented an extension of his treemap SLAM algorithm to 6DoF [11].

\subsection{Globally consistent range image alignment}

Besides the robotic community, computer vision researchers are interested in consistent alignment methods. Chen and Medioni [7] aimed at globally consistent range image alignment when introducing an incremental matching method, i.e., all new scans are registered against the so-called metascan, which is the union of the previously acquired and registered scans. This method does not spread out the error and is order dependent.

Bergevin et al. [4], Benjemaa and Schmitt [2,3], and Pulli [25] present iterative approaches. Based on networks representing overlapping parts of images, they use the ICP 
algorithm for computing transformations that are applied after all correspondences between all views have been found. However, the focus of research is mainly 3D modeling of small objects using a stationary 3D scanner and a turntable; therefore, the used networks consist mainly of one loop [25]. A probabilistic approach was proposed by Williams et al. [38], where each scan point is assigned a Gaussian distribution in order to model the statistical errors made by laser scanners. This causes high computation time due to the large amount of data in practice. Krishnan et al. [19] presented a global registration algorithm that minimizes the global error function by optimization on the manifold of $3 \mathrm{D}$ rotation matrices.

\section{Algorithm overview}

In the following the extension to $6 \mathrm{DoF}$ data of the global consistent scan matching approach by $\mathrm{Lu}$ and Milios is described. The scan matching process is outlined in Fig. 1.

All scans are registered sequentially using the ICP algorithm until convergence. To this end, the odometry of newly acquired scans is extrapolated to $6 \mathrm{DoF}$ using registration matrices of previously registered scans. We are using a left-handed coordinate system, i.e., the $y$ coordinate represents elevation. Then, the change of the robot pose $\Delta \mathbf{P}$, given the odometry information $\left(x_{n}^{\text {odo }}, z_{n}^{\text {odo }}, \theta_{y, n}^{\text {odo }}\right),\left(x_{n+1}^{\text {odo }}, z_{n+1}^{\text {odo }}, \theta_{y, n+1}^{\text {odo }}\right)$ and the registration matrix $\mathbf{R}\left(\theta_{x, n}, \theta_{y, n}, \theta_{z, n}\right)$, is calculated by solving:

$$
\begin{aligned}
& \left(\begin{array}{c}
x_{n+1}^{\text {odo }} \\
0 \\
z_{n+1}^{\text {odo }} \\
0 \\
\theta_{y, n+1}^{\text {odo }} \\
0
\end{array}\right)=\left(\begin{array}{c}
x_{n}^{\text {odo }} \\
0 \\
z_{n}^{\text {odo }} \\
0 \\
\theta_{y, n}^{\text {odo }} \\
0
\end{array}\right)+\left(\begin{array}{cc}
\mathbf{R}\left(\theta_{x, n}, \theta_{y, n}, \theta_{z, n}\right) & \mathbf{0} \\
\hline & \mathbf{0} \\
I_{3}
\end{array}\right) \\
& \underbrace{\left(\begin{array}{c}
\Delta x_{n+1} \\
\Delta y_{n+1} \\
\Delta z_{n+1} \\
\Delta \theta_{x, n+1} \\
\Delta \theta_{y, n+1} \\
\Delta \theta_{z, n+1}
\end{array}\right)}_{\Delta \mathbf{P}}
\end{aligned}
$$

Therefore, calculating $\Delta \mathbf{P}$ requires a matrix inversion. Finally, the $6 \mathrm{D}$ pose $\mathbf{P}_{n+1}$ is calculated by

$\mathbf{P}_{n+1}=\Delta \mathbf{P} \cdot \mathbf{P}_{n}$

using the poses' matrix representations.

Once a loop is detected, i.e., the distance between the poses of two scans falls below a threshold, the adaptation of Lu/Milios style scan matching described in Section 6 is applied. For each iteration, a network of pose relations is built automatically. From the corresponding scans, a linear equation system representing distance measurements is built and solved, resulting in optimized pose estimations. When the sum of all distances is below a threshold, new data is acquired and registered using ICP.

\section{3D scan matching}

We use the well-known iterative closest points (ICP) algorithm [5] to calculate the transformation while the robot is acquiring a sequence of $3 \mathrm{D}$ scans. ICP calculates the point correspondences iteratively. In each iteration step, the algorithm selects the closest points as correspondences and calculates the transformation $(\mathbf{R}, \mathbf{t})$ for minimizing the equation

$E(\mathbf{R}, \mathbf{t})=\sum_{i=1}^{N_{m}} \sum_{j=1}^{N_{d}} w_{i, j}\left\|\mathbf{m}_{i}-\left(\mathbf{R d}_{j}+\mathbf{t}\right)\right\|^{2}$,

where $N_{m}$ and $N_{d}$, are the numbers of points in the model set $M$ or data set $D$, respectively, and $w_{j, i}$ are the weights for a point match. The weights are assigned as follows: $w_{i, j}=$ 1 , if $\mathbf{m}_{i}$ is the closest point to $\mathbf{d}_{j}$ within a close limit, $w_{i, j}=0$ otherwise. The assumption is that in the last iteration the point correspondences are correct. In each iteration, the transformation is calculated by the quaternion based method of Horn [16].

To digitalize environments without occlusions, multiple 3D scans have to be registered. Consider a robot traveling along a path, and traversing $n+1$ poses $V_{0}, \ldots, V_{n}$. A straightforward method for aligning several $3 \mathrm{D}$ scans taken from $V_{0}, \ldots, V_{n}$ is pairwise ICP, i.e., matching the scan taken from pose $V_{1}$ against the scan from pose $V_{0}$, matching the scan taken from $V_{2}$ against the scan from pose $V_{1}$, and so on.

\section{Loop closing}

Pairwise ICP improves the robot pose estimates, but registration errors sum up. SLAM algorithms use loop closing to bound this error. If two estimated robot poses $V_{i}$ and $V_{j}$ are close enough, i.e., their distance falls below a threshold (here: $5 \mathrm{~m}$ ), we assume these scans overlap and are matchable. To a graph, initially containing the sequence of all poses $\left(V_{0}, V_{1}\right),\left(V_{1}, V_{2}\right), \ldots,\left(V_{n-1}, V_{n}\right)$, the edge $\left(V_{i}, V_{j}\right)$ is added.

While processing the scans with pairwise ICP, we detect closed loops using this simple distance criterion. Once detected, a 6D graph optimization algorithm for global relaxation based on the method of Lu and Milios [20] is employed, namely, Lu and Milios style SLAM (LUM). We extended this variant of GraphSLAM to 6DoF, as described in the next section.

If this simple loop closing strategy fails, the resulting map is incorrect. We accept this drawback, since a multi hypothesis approach with $6 \mathrm{DoF}$ is currently not tractable. In [40] we give a quantitative performance evaluation and an in depth analysis of the loop closing method.

\section{Global relaxation}

\subsection{Problem formulation}

Consider a robot traveling along a path, and traversing the $n+1$ poses $V_{0}, \ldots, V_{n}$. At each pose $V_{i}$, the robot stops to take a laser scan of its environment. By matching two scans made at two different poses, we acquire a set of neighbor relations between these poses. In the resulting network, nodes represent 


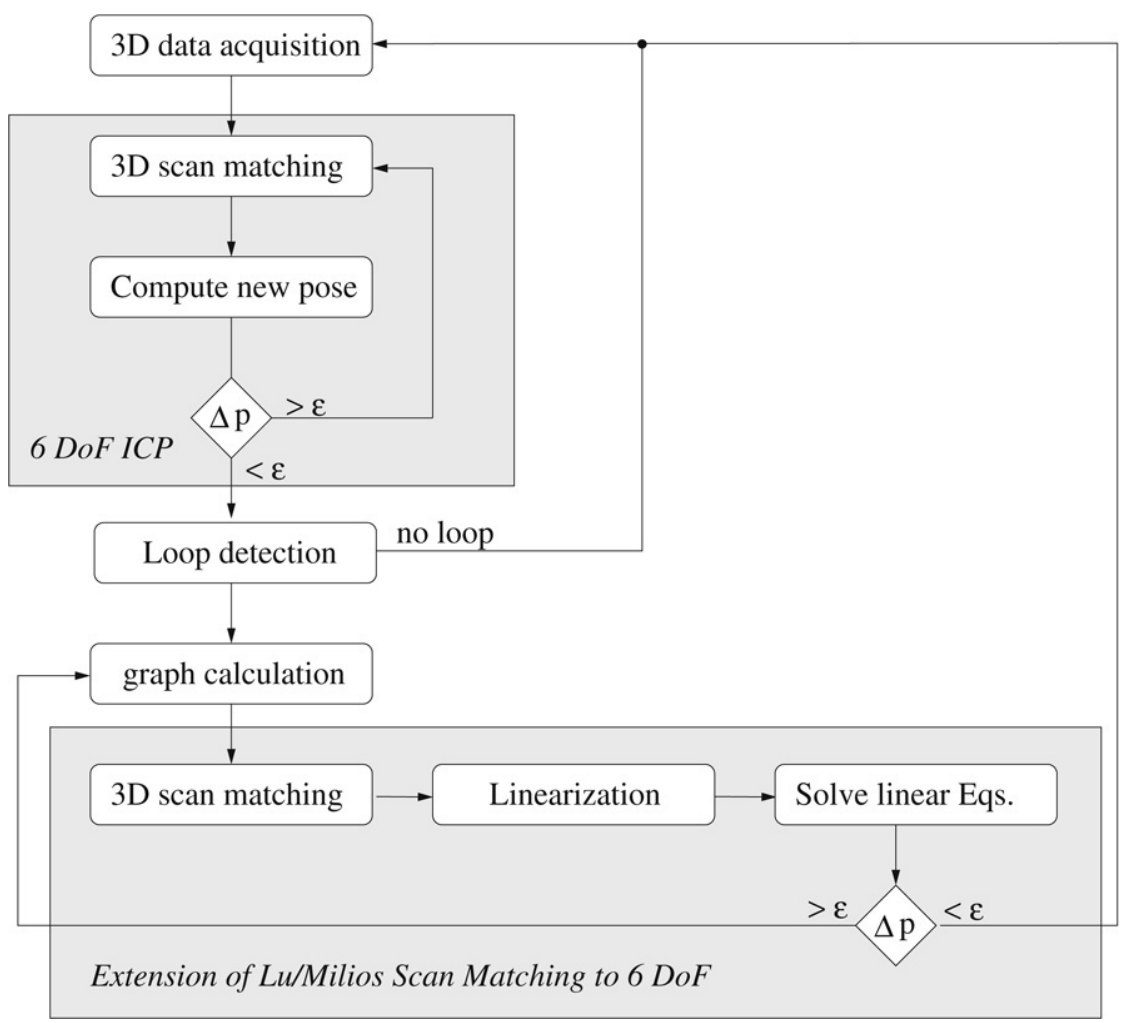

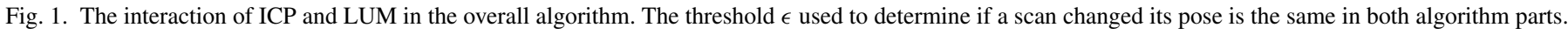

poses, and edges neighbor relations between them. Given such a network with $n+1$ graph nodes $X_{0}, \ldots, X_{n}$ and the directed edges $D_{i, j}$, we want to estimate optimally all poses to build a consistent map of the environment. For simplification, the measurement equation is assumed to be linear:

$D_{i, j}=X_{i}-X_{j}$.

The observation $\bar{D}_{i, j}$ of the true underlying difference is modeled as $\bar{D}_{i, j}=D_{i, j}+\Delta D_{i, j}$ where $\Delta D_{i, j}$ is a Gaussian distributed error with zero mean and a covariance matrix $C_{i, j}$, that is assumed to be known.

Maximum likelihood estimation is used to approximate the optimal poses $X_{i}$. Under the assumption that all errors in the observations are Gaussian and independently distributed, maximizing the probability of all $D_{i, j}$, given their observations $\bar{D}_{i, j}$, is equivalent to minimizing the following Mahalanobis distance:

$\mathbf{W}=\sum_{(i, j)}\left(D_{i, j}-\bar{D}_{i, j}\right)^{\mathrm{T}} C_{i, j}^{-1}\left(D_{i, j}-\bar{D}_{i, j}\right)$

\subsection{Solution as given by Lu and Milios}

We consider the simple linear case of the estimation problem. Without loss of generality we assume that the network is fully connected, i.e., each pair of nodes $X_{i}, X_{j}$ is connected by a link $D_{i, j}$. In the case of a missing link $D_{i, j}$ we set the corresponding $C_{i, j}^{-1}$ to 0 . Eq. (1) unfolds:

$\mathbf{W}=\sum_{(0 \leq i<j \leq n)}\left(X_{i}-X_{j}-\bar{D}_{i, j}\right)^{\mathrm{T}} C_{i, j}^{-1}\left(X_{i}-X_{j}-\bar{D}_{i, j}\right)$.
To minimize Eq. (2), a coordinate system is defined by setting one node as a reference point. Setting $X_{0}=(0,0,0)$, the $n$ free nodes $X_{1}, \ldots, X_{n}$ denote the poses relative to $X_{0}$.

Using the signed incidence matrix $\mathbf{H}$, the concatenated measurement equation $\mathbf{D}$ is written as

$\mathbf{D}=\mathbf{H X}$

with the concatenation $\mathbf{X}$ of $X_{1}$ to $X_{n}$. The Mahalanobis distance Eq. (1) can be written as:

$\mathbf{W}=(\overline{\mathbf{D}}-\mathbf{H X})^{\mathrm{T}} \mathbf{C}^{-1}(\overline{\mathbf{D}}-\mathbf{H X})$.

The concatenation of all observations $\bar{D}_{i, j}$ forms the vector $\overline{\mathbf{D}}$, while $\mathbf{C}$ is a block-diagonal matrix comprised of $C_{i, j}$ as submatrices. The solution $\mathbf{X}$ that minimizes the Eq. (2), and its covariance $\mathbf{C}_{\mathbf{X}}$ are given by

$$
\mathbf{X}=\left(\mathbf{H}^{\mathrm{T}} \mathbf{C}^{-1} \mathbf{H}\right)^{-1} \mathbf{H}^{\mathrm{T}} \mathbf{C}^{-1} \overline{\mathbf{D}}
$$$$
\mathbf{C}_{\mathbf{X}}=\left(\mathbf{H}^{\mathrm{T}} \mathbf{C}^{-1} \mathbf{H}\right)^{-1} \text {. }
$$

The matrix $\mathbf{G}=\mathbf{H}^{\mathrm{T}} \mathbf{C}^{-1} \mathbf{H}$ and the vector $\mathbf{B}=\mathbf{H}^{\mathrm{T}} \mathbf{C}^{-1} \overline{\mathbf{D}}$ simplify the notation of the solution. $\mathbf{G}$ consists of submatrices

$G_{i, j}= \begin{cases}\sum_{j=0}^{n} C_{i, j}^{-1} & (i=j) \\ C_{i, j}^{-1} & (i \neq j) .\end{cases}$

The entries of $\mathbf{B}$ are obtained by:

$B_{i}=\sum_{\substack{j=0 \\ j \neq i}}^{n} C_{i, j}^{-1} \bar{D}_{i, j}$ 
Solving the linear optimal estimation problem (3) is equivalent to solving the following linear equation system:

$\mathbf{G X}=\mathbf{B}$

\subsection{The extension to six degrees of freedom}

To apply the above solution to mobile robots, it is necessary to linearize the pose difference equation Eq. (2). The $3 \mathrm{DoF}$ case, i.e., $(x, z, \theta)^{\mathrm{T}}$ poses, was solved by Lu and Milios [20]. In addition to pose relations from scan matching, Lu and Milios considered pose relations from odometry. Our algorithm derives relations for 6 DoF poses, i.e., $\left(x, y, z, \theta_{x}, \theta_{y}, \theta_{z}\right)^{\mathrm{T}}$, by matching data obtained by a $3 \mathrm{D}$ laser range finder. The challenges of the extension were:

The amount of data. A 3D laser range finder scans the environment with a large number of samples. Typical resolutions vary between 23168 and 360500 range points per 3D scan.

Linearization. Linearization of the rotation must regard the 3DoF. The rotation consists of the three Euler angles $\left(\theta_{x}, \theta_{y}, \theta_{z}\right)$, and the multiplication of the corresponding three rotation matrices results in the desired overall rotation. By linearizing the Euler angles, we enforce valid rotation matrices.

Solution space. The additional three DoF result in an exponentially larger solution space. The solution is computationally more complex.

We define a $6 \mathrm{D}$ pose relation as follows: Assume that a robot starts at the pose $V_{b}=\left(x_{b}, y_{b}, z_{b}, \theta_{x_{b}}, \theta_{y_{b}}, \theta_{z_{b}}\right)^{\mathrm{T}}$ and changes its pose by $D=\left(x, y, z, \theta_{x}, \theta_{y}, \theta_{z}\right)^{\mathrm{T}}$ relative to $V_{b}$, ending up at $V_{a}=\left(x_{a}, y_{a}, z_{a}, \theta_{x_{a}}, \theta_{y_{a}}, \theta_{z_{a}}\right)^{\mathrm{T}}$. The poses $V_{a}$ and $V_{b}$ are related by the compounding operation $V_{a}=V_{b} \oplus D$. Similarly, a $3 \mathrm{D}$ position vector $u=\left(x_{u}, y_{u}, z_{u}\right)$ is compounded with the pose $V_{b}$ by $u^{\prime}=V_{b} \oplus u$ :

$x_{u}^{\prime}=x_{b}-z_{u} \sin \theta_{y_{b}}+\cos \theta_{y_{b}}\left(x_{u} \cos \theta_{z_{b}}-y_{u} \sin \theta_{z_{b}}\right)$

$y_{u}^{\prime}=y_{b}+z_{u} \cos \theta_{y_{b}} \sin \theta_{x_{b}}+\cos \theta_{x_{b}}\left(y_{u} \cos \theta_{z_{b}}+x_{u} \sin \theta_{z_{b}}\right)$ $+\sin \theta_{x_{b}} \sin \theta_{y_{b}}\left(x_{u} \cos \theta_{z_{b}}-y_{u} \sin \theta_{z_{b}}\right)$

$z_{u}^{\prime}=z_{b}-\sin \theta_{x_{b}}\left(y_{u} \cos \theta_{z_{b}}+x_{u} \sin \theta_{z_{b}}\right)+\cos \theta_{x_{b}}\left(z_{u} \cos \theta_{y_{b}}\right.$ $\left.+\sin \theta_{y_{b}}\left(x_{u} \cos \theta_{z_{b}}-y_{u} \sin \theta_{z_{b}}\right)\right)$.

This operation is used to transform a non-oriented point (from the scanner data) from its local to the global coordinate system.

Scan matching computes a set of $m$ corresponding point pairs $u_{k}^{a}, u_{k}^{b}$ between two scans, each representing a single physical point. The positional error made by identifying these two points in different scans, is described by:

$$
\begin{aligned}
F_{a b}\left(V_{a}, V_{b}\right) & =\sum_{k=1}^{m}\left\|V_{a} \oplus u_{k}^{a}-V_{b} \oplus u_{k}^{b}\right\|^{2} \\
& =\sum_{k=1}^{m}\left\|\left(V_{a} \ominus V_{b}\right) \oplus u_{k}^{a}-u_{k}^{b}\right\|^{2} .
\end{aligned}
$$

Table 1

\begin{tabular}{|c|c|c|}
\hline \multirow[t]{2}{*}{ Sensor data } & \multicolumn{2}{|c|}{ Dimensionality of pose representation } \\
\hline & 3D & $6 \mathrm{D}$ \\
\hline $2 \mathrm{D}$ & $\begin{array}{l}\text { Planar 2D mapping (2.2) } \\
\text { 2D mapping of planar sonar } \\
\text { and laser scans. }\end{array}$ & $\begin{array}{l}\text { Slice-wise } 6 \text { D SLAM (2.4) } \\
\text { mapping using a precise } \\
\text { localization, considering the } \\
x, y, z \text {-position and the roll, } \\
\text { yaw and pitch angle. }\end{array}$ \\
\hline $3 \mathrm{D}$ & $\begin{array}{l}\text { Planar 3D mapping (2.3) } \\
\text { 3D mapping using a planar } \\
\text { localization method and, } \\
\text { e.g., an upward looking } \\
\text { laser scanner or 3D } \\
\text { scanner. }\end{array}$ & $\begin{array}{l}\text { Full 6D SLAM ( } 2.5) \\
\text { mapping using } 3 \mathrm{D} \text { laser } \\
\text { scanners or (stereo) cameras } \\
\text { with pose estimates } \\
\text { calculated from the sensor } \\
\text { data. }\end{array}$ \\
\hline
\end{tabular}

Overview of the dimensionality of SLAM approaches

Shaded portion: 2D maps. Non-shaded portion: 3D maps.

Based on these $m$ point pairs, the algorithm computes the matrices $\bar{D}_{i, j}$ and $C_{i, j}$ for solving Eq. (1). $\bar{D}_{i, j}$ is derived as follows:

Let $\bar{V}_{a}=\left(\bar{x}_{a}, \bar{y}_{a}, \bar{z}_{a}, \bar{\theta}_{x_{a}}, \bar{\theta}_{y_{a}}, \bar{\theta}_{z_{a}}\right)$ and $\bar{V}_{b}=\left(\bar{x}_{b}, \bar{y}_{b}, \bar{z}_{b}, \bar{\theta}_{x_{b}}\right.$, $\left.\bar{\theta}_{y_{b}}, \bar{\theta}_{z b}\right)$ be close estimates of $V_{a}$ and $V_{b}$. If the global coordinates of a pair of matching points $u_{k}=\left(x_{k}, y_{k}, z_{k}\right)$, then $\left(u_{k}^{a}, u_{k}^{b}\right)$ fulfill the equation

$u_{k} \approx V_{a} \oplus u_{k}^{a} \approx V_{b} \oplus u_{k}^{b}$.

For small errors $\Delta V_{a}=\bar{V}_{a}-V_{a}$ and $\Delta V_{b}=\bar{V}_{b}-V_{b}$, a Taylor expansion leads to:

$$
\begin{aligned}
\Delta Z_{k}= & V_{a} \oplus u_{k}^{a}-V_{b} \oplus u_{k}^{b}:=F_{k}\left(V_{a}, V_{b}\right) \\
\approx & F_{k}\left(\bar{V}_{a}, \bar{V}_{b}\right)-\left[\nabla_{\bar{V}_{a}}\left(F_{k}\left(\bar{V}_{a}, \bar{V}_{b}\right)\right) \Delta V_{a}\right. \\
& \left.-\nabla_{\bar{V}_{b}}\left(F_{k}\left(\bar{V}_{a}, \bar{V}_{b}\right)\right) \Delta V_{b}\right] \\
= & \bar{V}_{a} \oplus u_{k}^{a}-\bar{V}_{b} \oplus u_{k}^{b}-\left[\nabla_{\bar{V}_{a}}\left(\bar{V}_{a} \oplus u_{k}^{a}\right) \Delta V_{a}\right. \\
& \left.-\nabla_{\bar{V}_{b}}\left(\bar{V}_{b} \oplus u_{k}^{b}\right) \Delta V_{b}\right]
\end{aligned}
$$

where $\nabla_{\bar{V}_{a}}\left(F_{k}\left(\bar{V}_{a}, \bar{V}_{b}\right)\right)$ is the gradient of the pose compounding operation. By matrix decomposition

$$
\begin{aligned}
& M_{k} H_{a}=\nabla_{\bar{V}_{a}}\left(F_{k}\left(\bar{V}_{a}, \bar{V}_{b}\right)\right) \\
& M_{k} H_{b}=\nabla_{\bar{V}_{b}}\left(F_{k}\left(\bar{V}_{a}, \bar{V}_{b}\right)\right),
\end{aligned}
$$

Eq. (9) simplifies to the equation given in Box I. This matrix decomposition and the derivation of $H_{a}, H_{b}$ is the crucial step in extending Lu and Milios style SLAM to 6DoF.

$D$ as defined by the second condition in Box $\mathrm{I}$ is the new linearized measurement equation. To calculate both $\bar{D}$ and $C_{D}$, the Eq. (8) is rewritten in matrix form

$F_{a b}(D) \approx(\mathbf{Z}-\mathbf{M} D)^{\mathrm{T}}(\mathbf{Z}-\mathbf{M} D)$.

$\mathbf{M}$ is the concatenated matrix consisting of all $M_{k}$ 's, and $\mathbf{Z}$ the concatenated vector consisting of all $Z_{k}$ 's. The vector $\bar{D}$ that minimizes $F_{a b}$ is given by

$\bar{D}=\left(\mathbf{M}^{\mathrm{T}} \mathbf{M}\right)^{-1} \mathbf{M}^{\mathrm{T}} \mathbf{Z}$.

Since minimizing $F_{a b}$ constitutes a least squares linear regression, we model the Gaussian distribution of the solution 


$$
\begin{aligned}
\Delta Z_{k} & \approx \bar{V}_{a} \oplus u_{k}^{a}-\bar{V}_{b} \oplus u_{k}^{b}-M_{k}\left[H_{a} \Delta V_{a}-H_{b} \Delta V_{b}\right] \\
& =\bar{Z}_{k}-M_{k} D
\end{aligned}
$$

with

$\bar{Z}_{k}=\bar{V}_{a} \oplus u_{k}^{a}-\bar{V}_{b} \oplus u_{k}^{b}$

$D=\left(H_{a} \Delta V_{a}-H_{b} \Delta V_{b}\right)$

$M_{k}=\left(\begin{array}{cccccc}1 & 0 & 0 & 0 & -y_{k} & -z_{k} \\ 0 & 1 & 0 & z_{k} & x_{k} & 0 \\ 0 & 0 & 1 & -y_{k} & 0 & x_{k}\end{array}\right)$

$H_{a}=\left(\begin{array}{cccccc}1 & 0 & 0 & 0 & \bar{z}_{a} \cos \left(\bar{\theta}_{x_{a}}\right)+\bar{y}_{a} \sin \left(\bar{\theta}_{x_{a}}\right) & \bar{y}_{a} \cos \left(\bar{\theta}_{x_{a}}\right) \cos \left(\bar{\theta}_{y_{a}}\right)-\bar{z}_{a} \cos \left(\bar{\theta}_{y_{a}}\right) \sin \left(\bar{\theta}_{x_{a}}\right) \\ 0 & 1 & 0 & -\bar{z}_{a} & -\bar{x}_{a} \sin \left(\bar{\theta}_{x_{a}}\right) & -\bar{x}_{a} \cos \left(\bar{\theta}_{x_{a}}\right) \cos \left(\bar{\theta}_{y_{a}}\right)-\bar{z}_{a} \sin \left(\bar{\theta}_{y_{a}}\right) \\ 0 & 0 & 1 & \bar{y}_{a} & -\bar{x}_{a} \cos \left(\bar{\theta}_{x_{a}}\right) & \bar{x}_{a} \cos \left(\bar{\theta}_{y_{a}}\right) \sin \left(\bar{\theta}_{x_{a}}\right)+\bar{y}_{a} \sin \left(\bar{\theta}_{y_{a}}\right) \\ 0 & 0 & 0 & 1 & 0 & \sin \left(\bar{\theta}_{y_{a}}\right) \\ 0 & 0 & 0 & 0 & \sin \left(\bar{\theta}_{x_{a}}\right) & \cos \left(\bar{\theta}_{x_{a}}\right) \cos \left(\bar{\theta}_{y_{a}}\right) \\ 0 & 0 & 0 & 0 & \cos \left(\bar{\theta}_{x_{a}}\right) & -\cos \left(\bar{\theta}_{y_{a}}\right) \sin \left(\bar{\theta}_{x_{a}}\right)\end{array}\right)$.

$H_{b}$ is given analogously.

Box I.

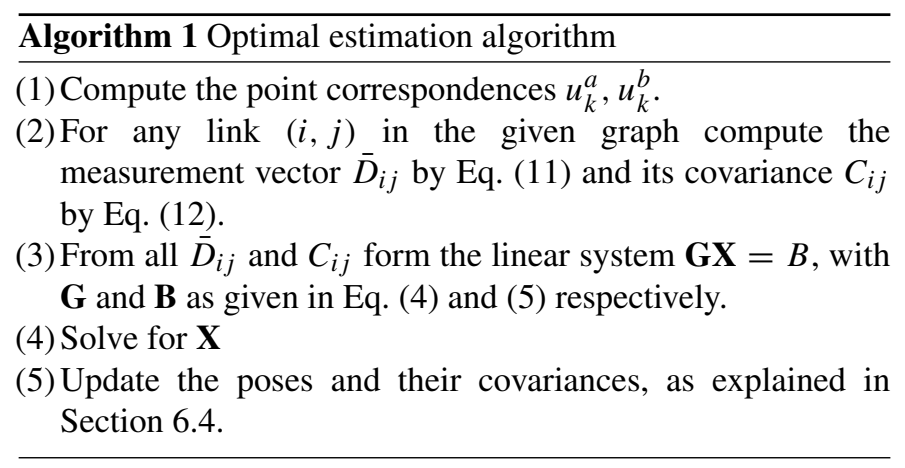

with mean $\bar{D}$ and standard covariance estimation

$C_{D}=s^{2}\left(\mathbf{M}^{\mathrm{T}} \mathbf{M}\right)$.

$s^{2}$ is the unbiased estimate of the covariance of the identically, independently distributed errors of $Z_{k}$, given by:

$s^{2}=(\mathbf{Z}-\mathbf{M} \bar{D})^{\mathrm{T}}(\mathbf{Z}-\mathbf{M} \bar{D}) /(2 m-3)=\frac{F_{a b}(\bar{D})}{2 m-3}$.

The error term $W_{a b}$ corresponding to our pose relation is defined by:

$W_{a b}=(\bar{D}-D)^{\mathrm{T}} C_{D}^{-1}(\bar{D}-D)$.

\subsection{Transforming the solution}

Solving the linear equation (6) leads to an optimal estimate of the new measurement equation of $D$ (second condition in Box I). To yield an optimal estimation of the robot poses, it is necessary to transform $D$. By this optimal estimation, a set of solutions $X_{i}=H_{i} \Delta V_{i}$ is computed, each corresponding to a node in the network.

Assuming that the reference pose $V_{0}=0$, the pose $V_{i}$ and its covariance $C_{i}$ are updated by: $\begin{aligned} V_{i} & =\bar{V}_{i}-H_{i}^{-1} X_{i}, \\ C_{i} & =\left(H_{i}^{-1}\right) C_{i}^{X}\left(H_{i}^{-1}\right)^{\mathrm{T}} .\end{aligned}$

If $V_{0}$ is nonzero, the solutions have to be transformed by:

$V_{i}^{\prime}=V_{0} \oplus V_{i}$

$C_{i}^{\prime}=K_{0} C_{i} K_{0}^{\mathrm{T}}$

where

$K_{0}=\left(\begin{array}{cc}R_{\theta_{x_{0}}, \theta_{y_{0}}, \theta_{z_{0}}} & 0 \\ 0 & I_{3}\end{array}\right)$

with a rotation matrix $R_{\theta_{x_{0}}, \theta_{y_{0}}, \theta_{z_{0}}}$.

\subsection{The algorithm}

The optimal estimation algorithm is given as Algorithm 1. Iterative execution of Algorithm 1 yields a successive improvement of the global pose estimation. Step 3 is sped up by component-wise computation of $\mathbf{G}$ and $\mathbf{B}$. The components $C_{i, j}^{-1}=\left(\mathbf{M}^{\mathrm{T}} \mathbf{M}\right) / s^{2}$ and $C_{i, j}^{-1} \bar{D}_{i, j}=\left(\mathbf{M}^{\mathrm{T}} \mathbf{Z}\right) / s^{2}$ are expanded into simple summations, as shown in Appendix B. The most expensive operation is solving the linear equation system $\mathbf{G X}=$ B. Since $\mathbf{G}$ is a positive definite, symmetric $6 n \times 6 n$ matrix, this is done by Cholesky decomposition in $\mathcal{O}\left(n^{3}\right)$.

\subsection{Performance issues}

The large amount of 3D data to be processed makes computing time an issue in globally consistent range scan matching. Our algorithm again benefits from the network structure. Each scan has to be aligned only to few neighbors in the graph. Compared to ICP metascan matching, LUM becomes more advantageous with increasing number of scans $n$. Our SLAM algorithm spends $\mathcal{O}\left(n^{3}\right)$ time on matrix computation. The matrices $\mathbf{B}$ and $\mathbf{G}$ are filled efficiently 


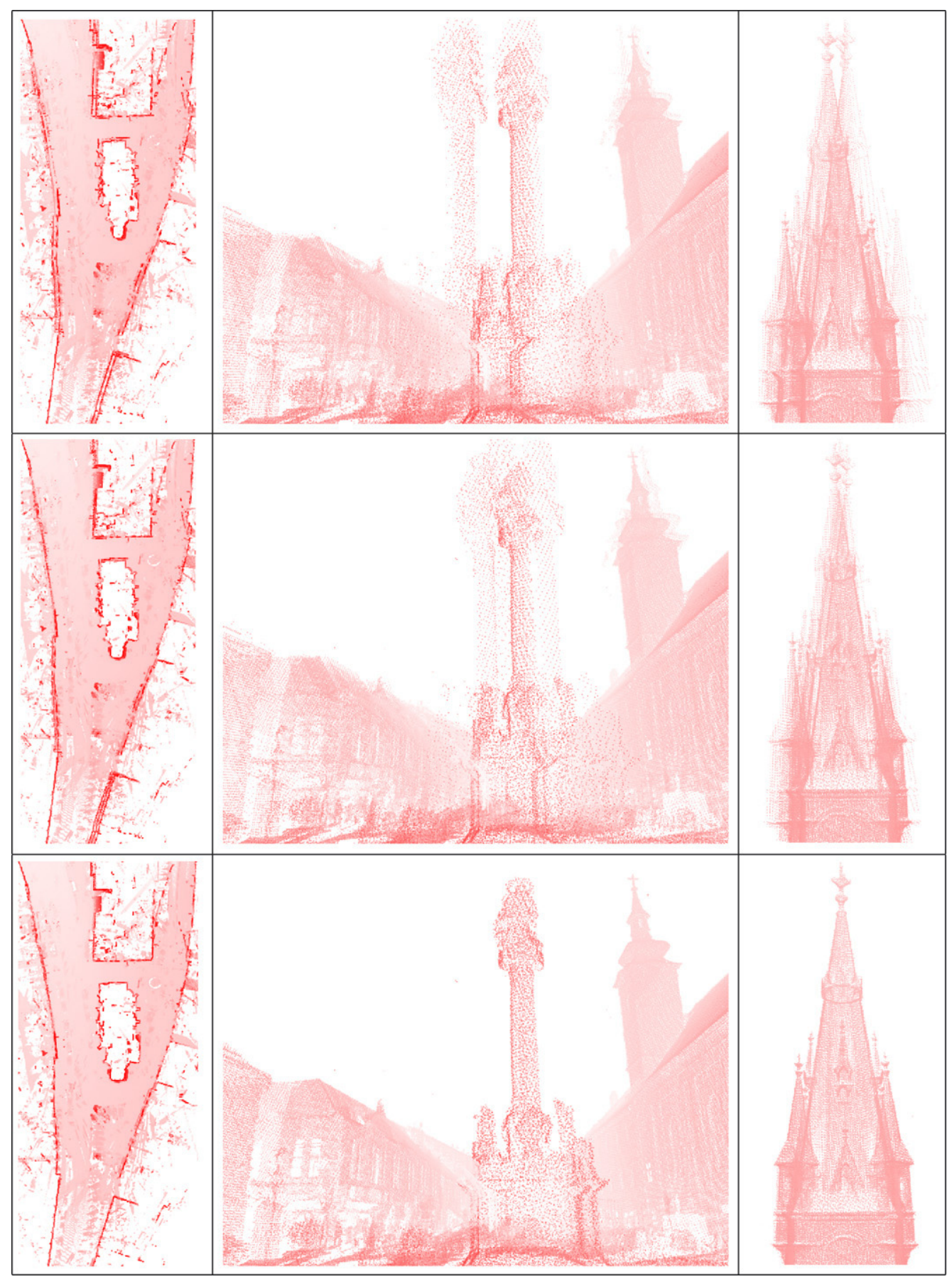

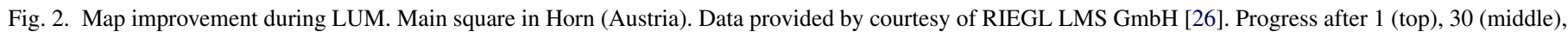

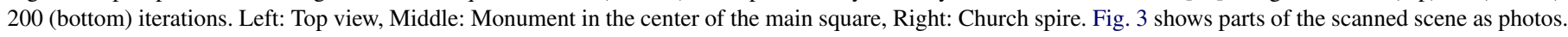

using simple additions (cf. Appendix B). However, calculating the corresponding points for a link needs $\mathcal{O}(N \log N)$, using standard nearest neighbor search methods, namely $k$-d trees. $N$ denotes the number of points per 3D scan, $n \ll N$. In all experiments the most computing time was spent in step 2 of Algorithm 1, e.g., $n<13, N<300000$ or $n<468, N<$ 18000 , respectively (cf. Section 7). Due to these performance issues, we presented several speed-ups for closest point computation in the scan matching context, i.e., approximate $k-\mathrm{d}$ tree search and cached $k$-d tree search $[22,23]$.

\subsection{Invertibility of $\mathbf{G}$}

The proposed algorithm depends on the invertibility of the matrix $\mathbf{G}$, which is the case if:

(1) All covariances are positive or negative definite, and:

(2) The pose graph is connected, i.e., there exist no two separate subgraphs.

The second condition is trivially met in practice, since all consecutive poses are linked. The inductive proof of the first 
Table 2

Position and orientation errors in the Horn data set

\begin{tabular}{cllll}
\hline 3D scan no. & Initial error $(\mathrm{m})$ (Eucl. distance) & Position error $(\mathrm{m})$ after registration & Initial error $(\mathrm{deg})\left(\theta_{x}, \theta_{y}, \theta_{z}\right)$ & Rotation error $(\mathrm{deg})$ after registration \\
\hline 1 & 0 & 0 & $(0.0,0.0,0.0)$ & $(0.0,0.0,0.0)$ \\
2 & 0.780 & 0.017 & $(0.0,4.0,0.0)$ & $(-0.0221,0.0177,-0.0116)$ \\
3 & 1.965 & 0.024 & $(0.0,4.0,0.0)$ & $(-0.0242,0.0257,-0.0236)$ \\
4 & 1.764 & 0.029 & $(0.0,2.0,0.0)$ & $(-0.0242,0.0228,-0.0200)$ \\
5 & 1.222 & 0.037 & $(0.0,5.0,0.0)$ & $(-0.0266,0.0298,-0.0339)$ \\
6 & 0.859 & 0.059 & $(0.0,1.0,0.0)$ & $(-0.0297,0.0183,0.0039)$ \\
7 & 2.151 & 0.052 & $(0.0,2.0,0.0)$ & $(0.0061,0.0195,-0.0269)$ \\
8 & 0.451 & 0.053 & $(0.0,1.0,0.0)$ & $(-0.0416,0.0173,0.0076)$ \\
9 & 1.023 & 0.069 & $(0.0,3.0,0.0)$ & $(-0.0690,0.0279,0.0170)$ \\
10 & 2.039 & 0.082 & $(0.0,1.0,0.0)$ & $(-0.0690,0.0165,0.0190)$ \\
11 & 1.654 & 0.059 & $(0.0,5.0,0.0)$ & $(-0.0988,0.0936,0.0040)$ \\
12 & 1.340 & 0.033 & $(0.0,2.0,0.0)$ & $(0.0030,0.0196,0.0410)$ \\
13 & 1.195 & 0.017 & $(0.0,5.0,0.0)$ & $(0.0022,0.0593,0.0510)$ \\
\hline
\end{tabular}

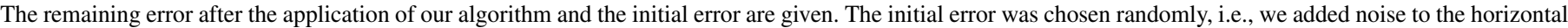
axis in position and to the vertical axis in orientation.

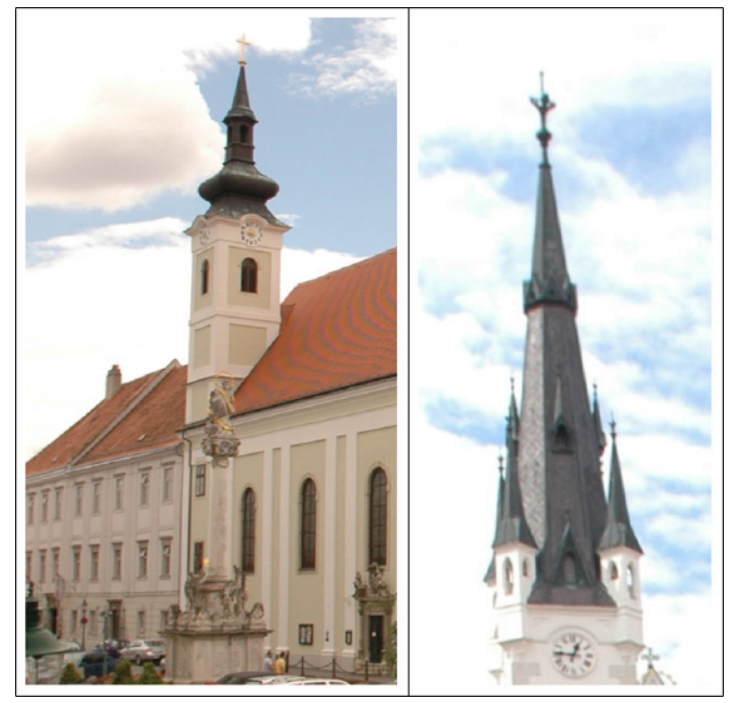

Fig. 3. Photos showing the scene presented in Fig. 2. Data provided by courtesy of RIEGL LMS GmbH [26]. Left: The right part of the middle detailed view. Right: The white-steepled St. Georg church.

condition over the number of nodes in the graph is given in Appendix A.

\section{Experiments and results}

The proposed algorithm has been tested in various experiments. In [6] we show by evaluating $2 \mathrm{D}$ laser range scans that the extension to three dimensions did not decrease the functionality of the algorithm in areas where it did work sufficiently well before. Furthermore 3D data was successfully aligned to ground truth when matching data from a planar indoor environment. Additionally, full functionality in all six DoFs was shown by use of data obtained from non-planar outdoor environments.

In this article, we first present an evaluation using high resolution 3D scans. The resulting poses are compared with ground truth. Second, highly connected data from a 3D environment is matched with an active loop closing algorithm. This leads to a network with many links that helps to diminish inconsistencies in the resulting map.

\subsection{Registration of high resolution outdoor 3D scans}

For this experiment we used a data set from the main square in Horn (Austria), consisting of 13 laser scans. Each scan is composed of 240000 to 300000 points. Scan matching with reduced points took $19 \mathrm{~min}$ ( $6 \mathrm{~min}$ for ICP and $13 \mathrm{~min}$ for LUM) until convergence, e.g. no scan was moved more than $0.5 \mathrm{~cm}$ in one iteration.

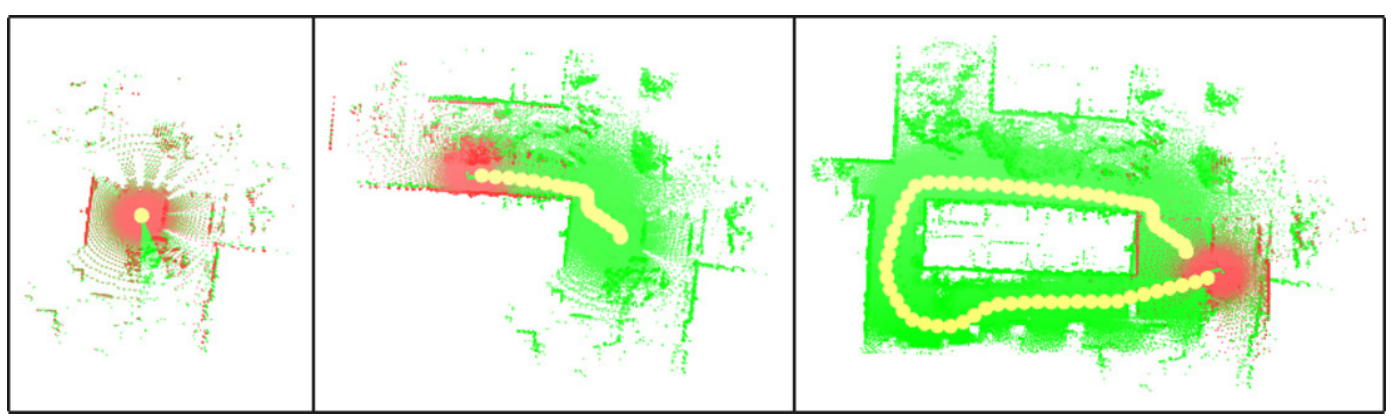

Fig. 4. Sequential ICP registration, from start to first loop detection, in the Hannover data set. 


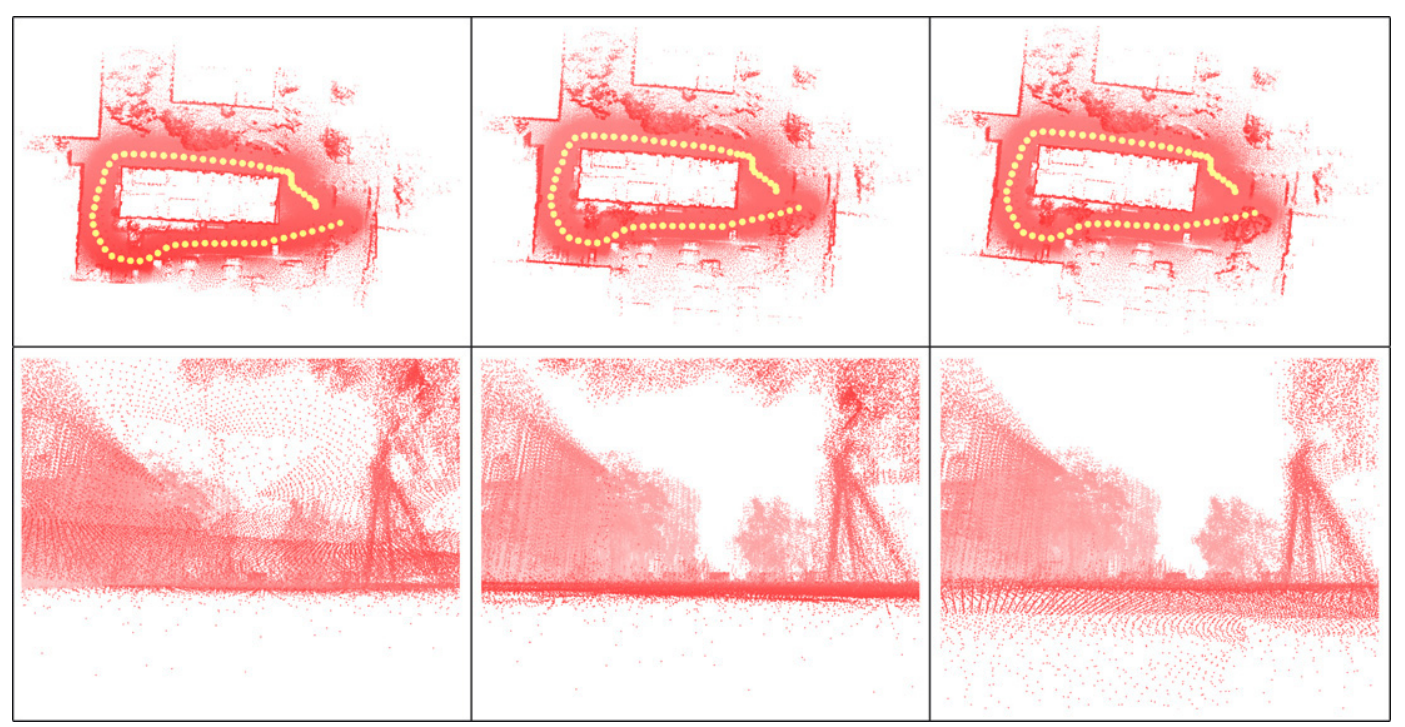

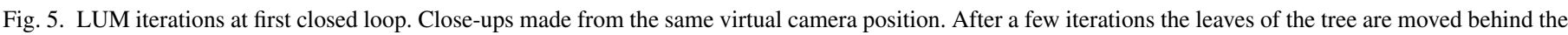
camera position (bottom). Left: 1, Middle: 10, Right: 77 iterations.

Parts of the resulting map can be seen in Fig. 2 (see http://kos.informatik.uni-osnabrueck.de/download/Riegl/ index.html for the whole map). From top to bottom, the number of iteration increases, and analogously the precision of the map. Qualitatively, no inconsistencies remain in the bottommost images.

For quantitative results, Table 2 shows the drastic reduction of errors compared to ground truth, acquired by manual alignment using retro-reflective targets of known coordinates [26], during scan matching. Pose errors are equally reduced for each laser scan, without any accumulation of errors. This holds not only for position errors but also for rotation errors, which pose the most significant challenge in 6D SLAM.

\subsection{Registration with dynamic network construction}

Detecting loops in sets of data helps build globally consistent maps, because it facilitates distributing larger errors over all scans. Each pose relation gives additional information for improving the calculation of optimal pose estimations. After loop detection, we automatically determine the pose network, using simple distance heuristics. By calculating the pose relations dynamically after each iteration of LUM, we obtain optimized poses, leading iteratively to more accurate networks. Scans that converge towards each other result in new pose relations, while connections between diverging scans disappear.

This is demonstrated on a set of 468 laser scans from a robot run at the Leibniz Universität Hannover while driving a distance of ca. $750 \mathrm{~m}$. Each scan consists of 14000 to 18000 scan points. The convergence limit was set to $0.1 \mathrm{~cm}$ movement per pose. Neighbor relations are established between all scans within a distance of less than $7.5 \mathrm{~m}$.

Figs. 4-8 show the maps after particular steps of scan matching. First, scans are registered iteratively using the ICP algorithm, generating the maps shown in Fig. 4. Once a loop is detected, LUM is used to achieve global consistency (cf. Fig. 5).
Fig. 6 shows the map after LUM at the second, third and fourth closed loops and Fig. 8 the final map. While matching the fourth loop, the robot path merges through recomputation of the graph, as shown in Fig. 7.

Ground truth for this data set is not available, therefore no comparison of our final 3D map to a reference 3D model is possible. Wulf et al. developed a method to benchmark our mapping algorithm using Monte Carlo localization in 2D reference maps from the German Land Registry office [40]. Using this novel benchmarking method on a similar data set they demonstrated that our algorithm maps the areas where the loops are closed with higher precision than the remaining parts.

\section{Summary}

One very active research area in robotics is mapping environments by matching point clouds collected by laser scanners. Popular techniques for 3D scan matching are based on minimizing the distances between point pairs detected in two corresponding range scans. Errors in laser scan data and imprecise matching methods lead to accumulated errors in the progress of building large maps, causing inconsistencies in regions where loops are closed.

This paper has presented a technique of matching laser scans globally consistently. Since a global error function is minimized in our approach, it avoids the common problems of sequential matching strategies.

The algorithm copes with the difficulties posed by the $6 \mathrm{DoF}$ and the large amount of data in a fast and robust manner. The instabilities reported in [31] for 2D scans and 3D poses did not occur in the 3D scan/6D pose case.

\section{Acknowledgments}

The authors would like to acknowledge Nikolaus Studnicka (RIEGL Laser Measurement Systems GmbH, Horn) and 


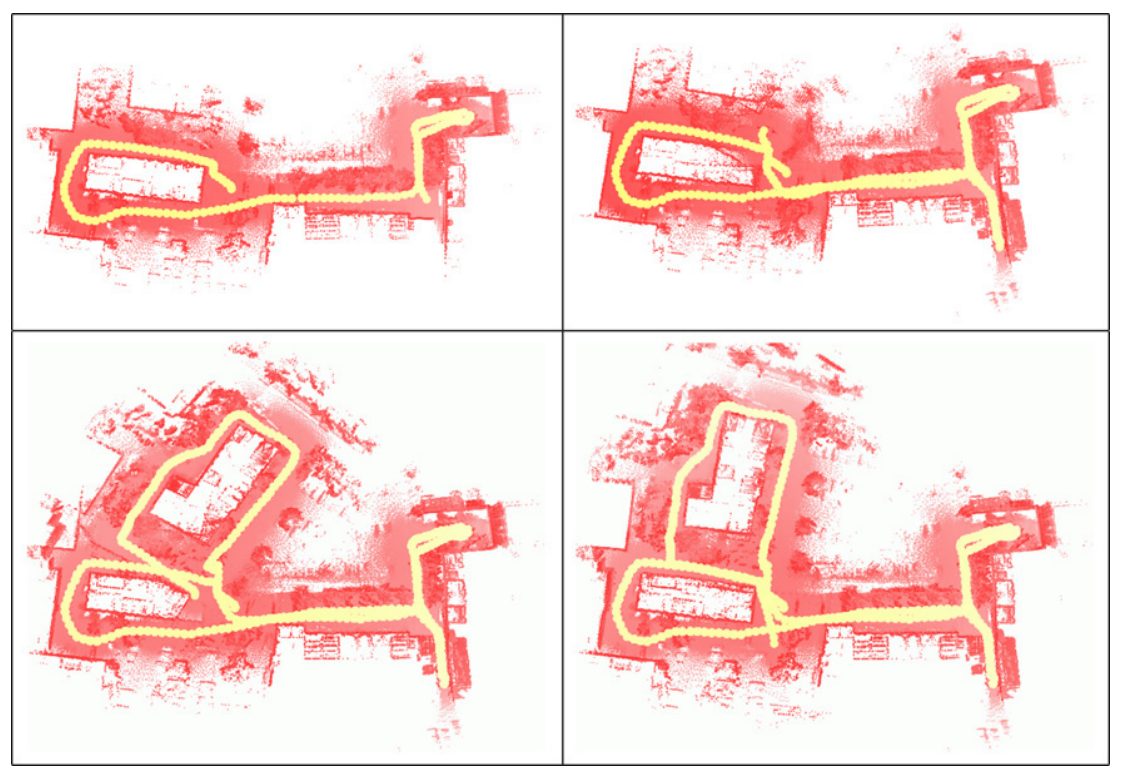

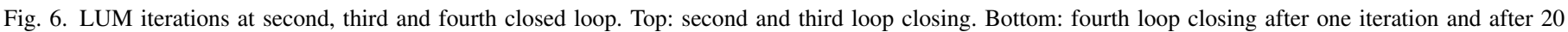

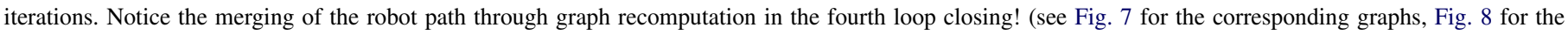
resulting map).

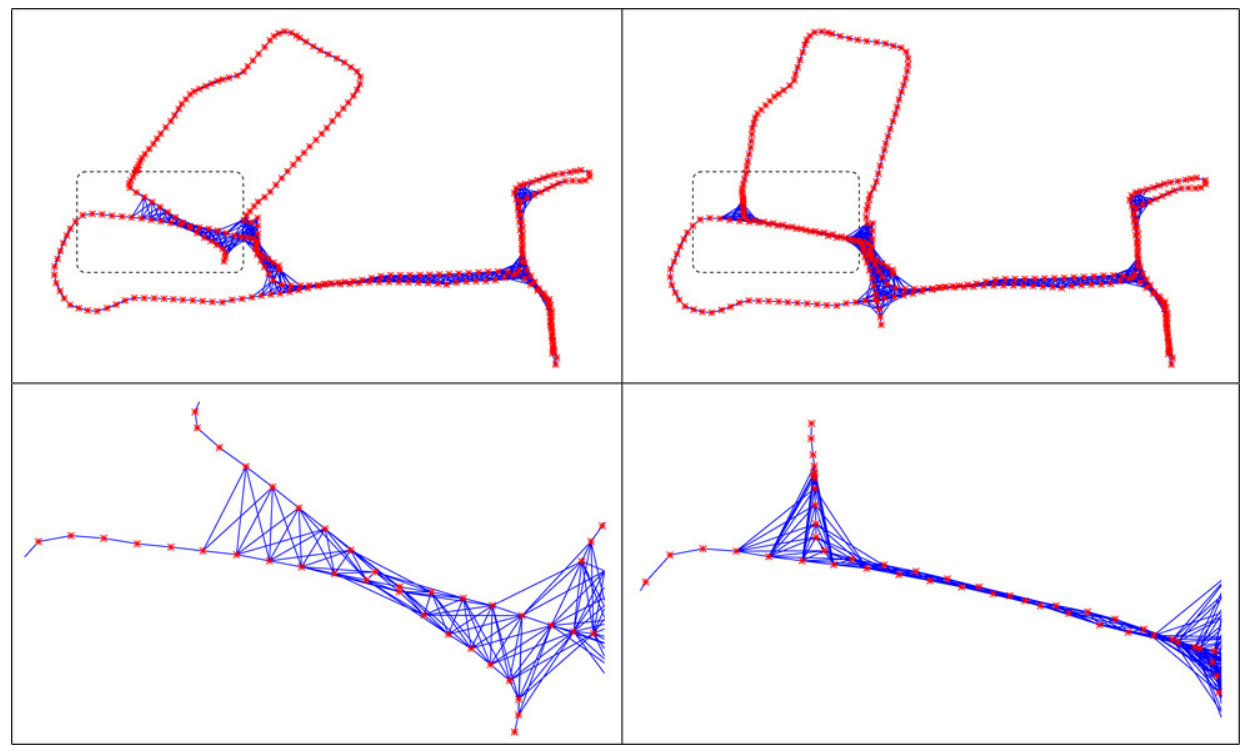

Fig. 7. Top: Graphs corresponding to the two alignments of Fig. 6 (Bottom). Bottom: Zoom into the boxed areas.

Oliver Wulf, Bernardo Wagner (Leibniz Universität Hannover) for providing the data sets. Further thanks to Evangelos E. Milios (Dalhousie University), Sebastian Thrun (Stanford University) and Szymon Rusinkiewicz (Princeton University) for answering questions about GraphSLAM methods.

\section{Appendix A. Proof of invertibility}

The algorithm described in this paper is based on the inversion of matrix $\mathbf{G}$. We prove that $\mathbf{G}$ is positive definite, and therefore invertible, using induction. In order to simplify the proof, we show that changing the reference pose does not change the positive definiteness of $\mathbf{G}$. Without loss of generality, $\mathbf{G}$ is a positive definite matrix of the form (4), with the reference node $X_{0}$. Switching the reference node to $X_{i}$ results in the matrix $\mathbf{G}^{\prime}$. These two matrices are related by

$\mathbf{G}^{\prime}=\mathbf{I}_{i} \mathbf{G}$

where $\mathbf{I}_{i}$ is an identity matrix of size $(d n \times d n)$ with a row of negative $(d \times d)$ identity matrices of the form:

$\mathbf{I}_{i}=\left(\begin{array}{ccc}I_{d(i-1)} & 0 & 0 \\ -I_{d} & \ldots & -I_{d} \\ 0 & 0 & I_{d(n-i+1)}\end{array}\right)$.

Multiplication with $\mathbf{I}_{i}$ corresponds to replacing the submatrices at $(i, j)$ with the negative sum of all submatrices at row $j$. Since $\mathbf{I}_{i}$ is invertible, $\mathbf{G}^{\prime}$ remains positive definite. 


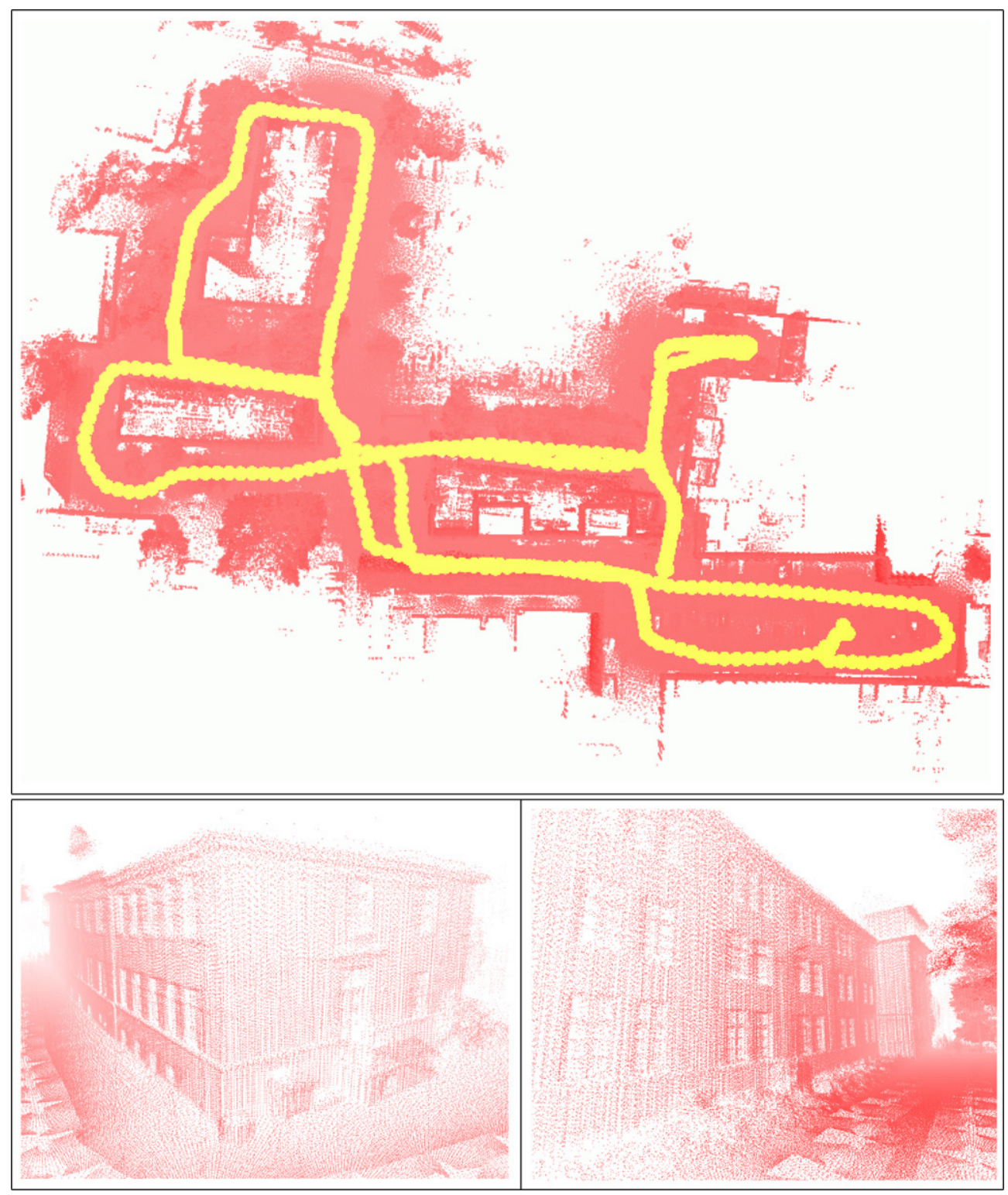

Fig. 8. Final map. Top: Top view including the final trajectory. Bottom: Two details rendered from the Hannover scene.

Induction base $k=n$ : Assuming a graph with $n+1$ nodes and $n$ links. The matrix $\mathbf{G}$ is transformed into the block diagonal matrix $\mathbf{G}^{\prime}$, composed of covariance matrices by

\section{$\mathbf{G}^{\prime}=\mathbf{I}_{\mathbf{D}} \mathbf{G} \mathbf{I}_{\mathbf{D}}^{T}$,}

with an upper-right triangular matrix $\mathbf{I}_{\mathbf{D}}$ of $d$-dimensional identity matrices

$\mathbf{I}_{\mathbf{D}}=\left(\begin{array}{ccc}I_{d} & \ldots & I_{d} \\ & \ddots & \vdots \\ 0 & & I_{d}\end{array}\right)$.

Since $\mathbf{G}^{\prime}$ is given by

$G_{i, i}^{\prime}=C_{i-1, i}^{-1}$

$G_{i, j}^{\prime}=0 \quad(i \neq j)$ and all covariances are positive definite, $\mathbf{G}^{\prime}$ itself is positive definite. The same holds for $\mathbf{G}$, as $\mathbf{I}_{\mathbf{D}}$ is invertible.

Inductive step $k \rightarrow k+1$ : Let $\mathbf{G}$ be a positive definite matrix that corresponds to a graph with $n+1$ nodes and $k$ links. An additional link between the nodes $X_{i}$ and $X_{j}$ is inserted, with positive definite covariance $C_{i, j}$. Without restriction, $X_{i}$ is the reference node of the given graph, since the reference pose is arbitrary. Thus, the resulting matrix $\mathbf{G}^{\prime}$ is changed only at the $d \times d$ submatrix $G_{j, j}^{\prime}$ :

$G_{j, j}^{\prime}=G_{j, j}+C_{i, j}^{-1}$

If $C_{i, j}$ is positive definite, $\mathbf{G}^{*}$ is positive definite, too, iff

$\mathbf{X}^{\mathrm{T}} \mathbf{G}^{\prime} \mathbf{X}>0 \quad \mathbf{X} \in \mathbb{R}^{d \cdot n} \quad \mathbf{X} \neq 0$, 
which is equivalent to

$$
\sum_{k, l=1}^{n} X_{k}^{\mathrm{T}} G_{k, l}^{\prime} X_{l}>0
$$

where $X_{k}$ are the $d$-dimensional subvectors of $\mathbf{X}$. Expanding Eq. (A.1) to

$$
\begin{aligned}
\sum_{k, l=1}^{n} X_{k}^{\mathrm{T}} G_{k, l}^{\prime} X_{l} & =X_{j}^{\mathrm{T}} G_{j, j}^{\prime} X_{j}+\sum_{\substack{k, l=1 \\
k \neq j \neq l}}^{n} X_{k}^{\mathrm{T}} G_{k, l} X_{l} \\
& =X_{j}^{\mathrm{T}} C_{i, j}^{-1} X_{j}+\sum_{k, l=1}^{n} X_{k}^{\mathrm{T}} G_{k, l} X_{l} \\
& =X_{j}^{\mathrm{T}} C_{i, j}^{-1} X_{j}+\mathbf{X}^{\mathrm{T}} \mathbf{G} \mathbf{X}>0 .
\end{aligned}
$$

$\mathbf{G}^{\prime}$ is a positive definite matrix.

\section{Appendix B. Fast construction of the linear equation system}

To solve the linear equation system $\mathbf{G X}=\mathbf{B}$,

$C_{D}^{-1}=\left(\mathbf{M}^{\mathrm{T}} \mathbf{M}\right) / s^{2}$

$C_{D}^{-1} \bar{D}=\left(\mathbf{M}^{\mathrm{T}} \mathbf{Z}\right) / s^{2}$

are needed. To calculate these efficiently, summations are substituted for matrix multiplication by using the regularities in the matrix $\mathbf{M} . \mathbf{M}^{\mathrm{T}} \mathbf{M}$ is represented as a sum over all corresponding point pairs:

$$
\begin{aligned}
& \mathbf{M}^{\mathrm{T}} \mathbf{M} \\
& =\sum_{k=1}^{m}\left(\begin{array}{cccccc}
1 & 0 & 0 & 0 & -y_{k} & -z_{k} \\
0 & 1 & 0 & z_{k} & x_{k} & 0 \\
0 & 0 & 1 & -y_{k} & 0 & x_{k} \\
0 & z_{k} & -y_{k} & y_{k}^{2}+z_{k}^{2} & x_{k} z_{k} & -x_{k} y_{k} \\
-y_{k} & x_{k} & 0 & x_{k} z_{k} & y_{k}^{2}+x_{k}^{2} & y_{k} z_{k} \\
-z_{k} & 0 & x_{k} & -x_{k} y_{k} & y_{k} z_{k} & x_{k}^{2}+z_{k}^{2}
\end{array}\right) .
\end{aligned}
$$

Similarly, $\mathbf{M}^{\mathrm{T}} \mathbf{Z}$ is calculated as follows:

$$
\mathbf{M}^{\mathrm{T}} \mathbf{Z}=\sum_{k=0}^{m}\left(\begin{array}{c}
\Delta x_{k} \\
\Delta y_{k} \\
\Delta z_{k} \\
-z_{k} \cdot \Delta y_{k}+y_{k} \cdot \Delta z_{k} \\
-y_{k} \cdot \Delta x_{k}+x_{k} \cdot \Delta y_{k} \\
z_{k} \cdot \Delta x_{k}-x_{k} \cdot \Delta z_{k}
\end{array}\right)
$$

with

$$
\left(\begin{array}{c}
\Delta x_{k} \\
\Delta y_{k} \\
\Delta z_{k}
\end{array}\right)=\bar{Z}_{k}=\bar{V}_{a} \oplus u_{k}^{a}-\bar{V}_{b} \oplus u_{k}^{b}
$$

and an approximation for each point:

$$
\left(\begin{array}{l}
x_{k} \\
y_{k} \\
z_{k}
\end{array}\right)=u_{k} \approx\left(\bar{V}_{a} \oplus u_{k}^{a}+\bar{V}_{b} \oplus u_{k}^{b}\right) / 2 .
$$

Finally, $s^{2}$ is a simple summation using the observation of the linearized measurement equation $\bar{D}=\left(\mathbf{M}^{\mathrm{T}} \mathbf{M}\right)^{-1} \mathbf{M}^{\mathrm{T}} \mathbf{Z}$ :

$$
\begin{aligned}
s^{2}= & \sum_{k=0}^{m}\left[\left(\Delta x_{k}-\left(\bar{D}_{0}-y_{k} \cdot \bar{D}_{4}+z_{k} \cdot \bar{D}_{5}\right)\right)^{2}\right. \\
& +\left(\Delta y_{k}-\left(\bar{D}_{1}-z_{k} \cdot \bar{D}_{3}+x_{k} \cdot \bar{D}_{4}\right)\right)^{2} \\
& \left.+\left(\Delta z_{k}-\left(\bar{D}_{2}+y_{k} \cdot \bar{D}_{3}-x_{k} \cdot \bar{D}_{5}\right)\right)^{2}\right] .
\end{aligned}
$$

$\bar{D}_{i}$ denotes the $i$-th entry of the vector $\bar{D}$. Summation of $C_{D}^{-1}$ and $C_{D}^{-1} \bar{D}$ yields $\mathbf{B}$ and $\mathbf{G}$.

\section{References}

[1] P. Allen, I. Stamos, A. Gueorguiev, E. Gold, P. Blaer, AVENUE: Automated site modelling in urban environments, in: Proceedings of the Third International Conference on 3D Digital Imaging and Modeling, 3DIM '01, Quebec City, Canada, May 2001.

[2] R. Benjemaa, F. Schmitt, Fast global registration of 3D sampled surfaces using a multi-Z-buffer technique, in: Proceedings IEEE International Conference on Recent Advances in 3D Digital Imaging and Modeling, 3DIM '97, Ottawa, Canada, May 1997.

[3] R. Benjemaa, F. Schmitt, A solution for the registration of multiple 3D point sets using unit quaternions, Computer Vision - ECCV '98 2 (1998) 34-50.

[4] R. Bergevin, M. Soucy, H. Gagnon, D. Laurendeau, Towards a general multi-view registration technique, IEEE Transactions on Pattern Analysis and Machine Intelligence (PAMI) 18 (5) (1996) 540-547.

[5] P. Besl, N. McKay, A method for registration of 3-D shapes, IEEE Transactions on Pattern Analysis and Machine Intelligence 14 (2) (1992) 239-256.

[6] D. Borrmann, J. Elseberg, K. Lingemann, A. Nüchter, J. Hertzberg, Efficient extension of Lu and Milios SLAM to six degrees of freedom (in preparation)

[7] Y. Chen, G. Medioni, Object modelling by registration of multiple range images, Image and Vision Computing 10 (3) (1992) 145-155.

[8] D.M. Cole, P.M. Newman, Using laser range data for 3D SLAM in outdoor environments, in: Proceedings of the IEEE International Conference on Robotics and Automation, ICRA '06, Florida, USA, 2006.

[9] M.W.M.G. Dissanayake, P. Newman, S. Clark, H.F. Durrant-Whyte, M. Csorba, A solution to the simultaneous localization and map building (SLAM) problem, IEEE Transactions on Robotics and Automation 17 (3) (2001) 229-241.

[10] J. Folkesson, H. Christensen, Outdoor exploration and SLAM using a compressed filter, in: Proceedings of the IEEE International Conference on Robotics and Automation, ICRA '03, Taipei, Taiwan, September 2003, pp. 419-426.

[11] U. Frese, Efficient 6-DOF SLAM with treemap as a generic backend, in: Proceedings of the IEEE Internation Conference on Robotics and Automation, ICRA '07, Rome, Italy, April 2007.

[12] U. Frese, G. Hirzinger, Simultaneous localization and mapping - a discussion, in: Proceedings of the IJCAI Workshop on Reasoning with Uncertainty in Robotics, Seattle, USA, August 2001, pp. 17-26.

[13] C. Früh, A. Zakhor, 3D model generation for cities using aerial photographs and ground level laser scans, in: Proceedings of the Computer Vision and Pattern Recognition Conference, CVPR '01, Kauai, Hawaii, USA, December 2001.

[14] A. Georgiev, P.K. Allen, Localization methods for a mobile robot in urban environments, IEEE Transaction on Robotics and Automation (TRO) 20 (5) (2004) 851-864.

[15] M. Hebert, M. Deans, D. Huber, B. Nabbe, N. Vandapel, Progress in 3-D mapping and localization, in: Proceedings of the 9th International Symposium on Intelligent Robotic Systems, SIRS '01, Toulouse, France, July 2001

[16] B.K.P. Horn, Closed-form solution of absolute orientation using unit quaternions, Journal of the Optical Society of America A 4 (4) (1987) 629-642. 
[17] R. Katz, N. Melkumyan, J. Guivant, T. Bailey, J. Nieto, E. Nebot, Integrated sensing framework for 3D mapping in outdoor navigation, in: Proceedings of the IEEE/RSJ International Conference on Intelligent Robots and Systems, IROS '06, Beijing, China, October 2006.

[18] P. Kohlhepp, M. Walther, P. Steinhaus, Schritthaltende 3D-kartierung und lokalisierung für mobile Inspektionsroboter, in: R. Dillmann, H. Wörn, T. Gockel (Eds.), in: Proceedings of the Autonome Mobile Systeme 2003, 18. Fachgespraeche, December 2003.

[19] S. Krishnan, P.Y. Lee, J.B. Moore, S. Venkatasubramanian, Global registration of multiple 3D point sets via optimization on a manifold, in: Eurographics Symposium on Geometry Processing, 2000.

[20] F. Lu, E. Milios, Globally consistent range scan alignment for environment mapping, Autonomous Robots 4 (April) (1997) 333-349.

[21] M. Magnusson, T. Duckett, A comparison of 3D registration algorithms for autonomous underground mining vehicles, in: Proceedings of the Second European Conference on Mobile Robotics, ECMR '05, Ancona, Italy, September 2005, pp. 86-91

[22] A. Nüchter, K. Lingemann, J. Hertzberg, H. Surmann, 6D SLAM with approximate data associoation, in: Proceedings of the 12th IEEE International Conference on Advanced Robotics, ICAR '05, Seattle, USA, July 2005, pp. 242-249.

[23] A. Nüchter, K. Lingemann, J. Hertzberg, H. Surmann, 6D SLAM for 3D mapping outdoor environments, Journal of Field Robotics 24 (8-9) (2007).

[24] E. Olson, J. Leonard, S. Teller, Fast iterative alignment of pose graphs with poor initial estimates, in: Proceedings of the IEEE International Conference on Robotics and Automation, 2006.

[25] K. Pulli, Multiview registration for large data sets, in: Proceedings of the 2nd International Conference on 3D Digital Imaging and Modeling, 3DIM '99, Ottawa, Canada, October 1999, pp. 160-168.

[26] RIEGL Laser Measurement Systems GmbH, http://www.riegl.com.

[27] V. Sequeira, K. Ng, E. Wolfart, J. Goncalves, D. Hogg, Automated 3D reconstruction of interiors with multiple scan-views, in: Proceedings of SPIE, Electronic Imaging '99, The Society for Imaging Science and Technology/SPIE's 11th Annual Symposium, San Jose, CA, USA, January 1999.

[28] H. Surmann, A. Nüchter, K. Lingemann, J. Hertzberg, A 3D laser range finder for autonomous mobile robots, in: Proceedings of the 32nd International Symposium on Robotics, ISR '01, Seoul, Korea, April 2001.

[29] S. Thrun, Robotic mapping: A survey, CMU-CS-02-111, February 2002, School of Computer Science, Carnegie Mellon University, Pittsburgh, PA 15213.

[30] S. Thrun, W. Burgard, D. Fox, A probabilistic approach to concurrent mapping and localization for mobile robots, Machine Learning and Autonomous Robots 31 (5) (1997) 1-25.

[31] S. Thrun, W. Burgard, D. Fox, Probabilistic Robotics, MIT Press, 2005.

[32] S. Thrun, D. Fox, W. Burgard, A real-time algorithm for mobile robot mapping with application to multi robot and 3D mapping, in: Proceedings of the IEEE International Conference on Robotics and Automation, ICRA 00, San Francisco, CA, USA, April 2000

[33] S. Thrun, Y. Liu, D. Koller, A.Y. Ng, Z. Ghahramani, H.F. DurrantWhyte, Simultaneous localization and mapping with sparse extended information filters, Machine Learning and Autonomous Robots 23 (7-8) (2004) 693-716.

[34] S. Thrun, M. Montemerlo, A. Aron, Probabilistic terrain analysis for highspeed desert driving, in: Proceedings of Robotics: Science and Systems, Cambridge, USA, June 2006.

[35] R. Triebel, W. Burgard, Improving simultaneous localization and mapping in 3D using global constraints, in: Proceedings of the National Conference on Artificial Intelligence, AAAI '05, 2005.

[36] R. Triebel, P. Pfaff, W. Burgard, Multi-level surface maps for outdoor terrain mapping and loop closing, in: Proceedings of the International Conference on Intelligent Robots and Systems, IROS, 2006.

[37] J. Weingarten, R. Siegwart, EKF-based 3D SLAM for structured environment reconstruction, in: Proceedings of the IEEE/RSJ International Conference on Intelligent Robots and Systems, IROS '05, Edmonton, Alberta Canada, August 2005, pp. 2089-2094.

[38] J.A. Williams, M. Bennamoun, Multiple view 3D registration using statistical error models, Vision Modeling and Visualization (1999).
[39] O. Wulf, K.O. Arras, H.I. Christensen, B.A. Wagner, 2D mapping of cluttered indoor environments by means of 3D Perception, in: Proceedings of the IEEE International Conference on Robotics and Automation, ICRA '04, New Orleans, USA, April 2004, pp. 4204-4209.

[40] O. Wulf, A. Nüchter, J. Hertzberg, B.A. Wagner, Ground truth evaluation of large urban 6D SLAM, In: Proceedings of the IEEE/RSJ International Conference on Intelligent Robots and Systems, IROS '07, San Diego, USA, October 2007 (in press).

[41] H. Zhao, R. Shibasaki, Reconstructing textured CAD model of urban environment using vehicle-borne laser range scanners and line cameras, in: Second International Workshop on Computer Vision System, ICVS '01, Vancouver, Canada, 2001, pp. 284-295.

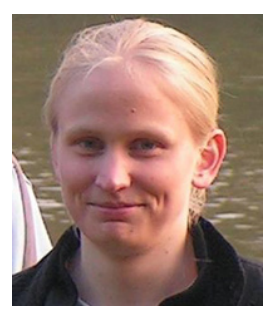

Dorit Borrmann received a B.Sc. degree in Mathematics/Computer Science from the University of Osnabrück in 2006. She continued her studies at graduate level there. In her research she focuses on 3D scan matching algorithms and their applications.

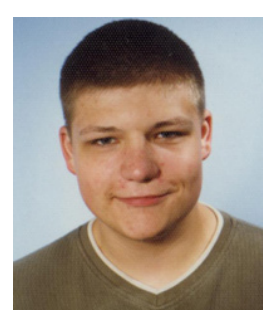

Jan Elseberg received a B.Sc. degree in Mathematics/Computer Science from the University of Osnabrück in 2006. He continued his studies at graduate level there. In his research he focuses on 3D scan matching algorithms and their applications.

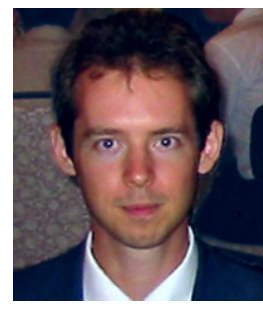

Kai Lingemann is a research associate at the University of Osnabrück. Past affiliations were with the Fraunhofer Institute for Autonomous Intelligent Systems (AIS, Sankt Augustin), University of Kyoto and University of Bonn, from which he received the diploma degree in computer science in 2004. His research interests include reliable robot control, 3D environment mapping, 3D vision, and laser scanning technologies.

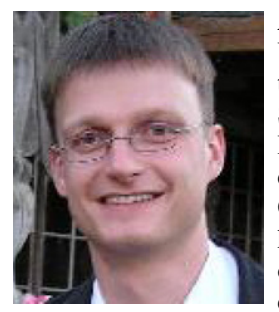

Andreas Nüchter is a research associate at the University of Osnabrück. Past affiliations were with the Fraunhofer Institute for Autonomous Intelligent Systems (AIS, Sankt Augustin) and University of Bonn, from which he received the diploma degree in computer science in 2002 (best paper award by the German Society of Informatics (GI) for his thesis). He holds a doctorate degree (Dr. rer. nat) from University of Bonn. His research interests include reliable robot control, 3D environment mapping, 3D vision, and laser scanning technologies, resulting in fast 3D scan matching algorithms that enable robots to map their environment in 3D using six degrees of freedom poses. The capabilities of these robotic SLAM approaches were demonstrated at RoboCup Rescue competitions, ELROB and several other events. He is a member of the GI and the IEEE.

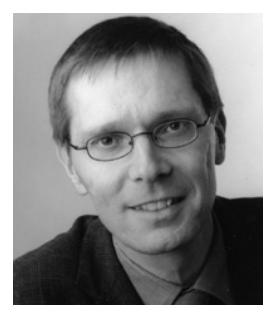

Joachim Hertzberg is a full professor for computer science at the University of Osnabrück, where he is heading the Knowledge-Based Systems lab and is currently the Dean of the school of Mathematics/Computer Science. He graduated in Computer Science (U. Bonn, 1982; Dr. rer. nat. 1986, U. Bonn; habilitation 1995, U. Hamburg). Former affiliations were with GMD and with Fraunhofer AIS in Sankt Augustin. His areas of scientific interest are Artificial Intelligence and Mobile Robotics, where he has contributed to action planning, robot localization and mapping, plan-based robot control, active sensing, robot control architectures, temporal reasoning, logical reasoning about action and change, constraint-based reasoning, and applications of these. In these areas, he has written or edited six books and published over 90 refereed or invited papers in books, journals or conferences. 